\title{
Dynamic Model Atmospheres of Cool Giants
}

\author{
Susanne Höfner ${ }^{1,2}$, Rita Gautschy-Loidl ${ }^{4}$, Bernhard Aringer ${ }^{3}$, Walter \\ Nowotny ${ }^{3}$, Josef Hron ${ }^{3}$, and Bernd Freytag ${ }^{1}$ \\ 1 Dept. of Astronomy \& Space Physics, Box 515, SE-75120 Uppsala, Sweden \\ 2 NORDITA, Blegdamsvej 17, DK-2100 Copenhagen, Denmark \\ 3 Inst. f. Astronomie, Türkenschanzstr. 17, A-1180 Vienna, Austria \\ 4 Froburgstr. 43, CH-4052 Basel, Switzerland
}

\begin{abstract}
Cool giant stars are highly dynamical objects, and complex micro-physical processes play an important role in their extended atmospheres and winds. The interpretation of observations, and in particular of high-resolution IR spectra, requires realistic self-consistent model atmospheres. Current dynamical models include rather detailed micro-physics, and the resulting synthetic spectra compare reasonably well with observations. A transition from qualitative to quantitative modelling is taking place at present. We give an overview of existing dynamical model atmospheres for AGB stars, discussing recent advances and current trends in modelling. When comparing synthetic spectra and other observable properties resulting from dynamical models with observations we focus on the near- and mid-IR wavelength range.
\end{abstract}

\section{Introduction}

Cool giant stars are prime targets for high-resolution infrared spectroscopy: these stars are luminous, emit most of their radiation at near- and mid-infrared wavelengths, and they have highly dynamical atmospheres due to convection and pulsation, leading to strong spectral variations. The coolest and most luminous of these objects also have slow, massive stellar winds. They represent an important but not so well-understood stage in the evolution of low- and intermediate mass stars, and they contribute significantly to the chemical enrichment of the interstellar medium. These are good reasons for studying cool giants but the interpretation of the observed spectra requires detailed and consistent model atmospheres for this type of stars.

Several of the very reasons that make pulsating asymptotic giant branch (AGB) stars interesting targets for IR spectroscopy, however, cause major challenges for realistic, self-consistent modelling of their dynamical atmospheres and circumstellar envelopes. Many interacting processes have to be taken into account simultaneously: propagating shock waves caused by stellar pulsation modify the structures of the atmospheres on local and global scales, leading to strong deviations from hydrostatic stratification. The radiative fields are dominated by molecular opacities or even by dust grains forming in the cool outer layers of the atmospheres. Important micro-physical processes like gas-phase chemistry and dust formation may be severely out of equilibrium.

Pioneering models for dynamic atmospheres of AGB stars date back more than two decades, but these models were severely restricted by the lack of sufficiently powerful computers and micro-physical input data. Only recently it 
has become possible to calculate consistent dynamic models which produce reasonably realistic synthetic spectra for a wide range of stellar parameters. This progress is of particular interest in the context of current instrumental developments in IR spectroscopy.

\section{Dynamic model atmospheres}

We begin this section with a definition of the term 'dynamic model atmospheres', in contrast to classical hydrostatic model atmospheres, stationary wind models, or pure circumstellar envelope models (which do not include the stellar atmosphere and wind acceleration region). The computational domain of such dynamic model atmospheres is a spherical shell, extending from an inner boundary below the stellar photosphere to an outer boundary which can be anywhere up to several tens of stellar radii above the photosphere, depending on whether the models have a stellar wind or not. The stellar pulsation is usually simulated by a variable inner boundary ('piston models') and outflow of matter is permitted at the outer boundary in the case of a stellar wind. Within this radial range, the time-dependent equations of hydrodynamics are solved, accounting for forces due to gravity, gas pressure and radiative acceleration of molecules and dust grains. Simultaneously, a more or less sophisticated treatment of radiative transfer is used to determine energy exchange. ${ }^{1}$ The results of the numerical simulations are consistent spatial structures of velocities, densities, temperatures and other physical quantities as a function of time. Furthermore, these models may give mass loss rates, dust-to-gas ratios and other stellar wind properties.

At present, four major 'strains' of dynamical models are in use. They share the basic ingredients given above but differ in the treatment of certain physical processes and the numerical methods. Listing them by their place of origin, their special features (and main references) can be summarised as follows:

- The Australian models (Wood [35], Bessel et al. [3], Hofmann et al. [12]) are strongly linked to pulsation models, featuring the most consistent description of the variable inner boundary available at present (cf. Sect. 4). They have been used to derive synthetic monochromatic radii and molecular line profiles to investigate the pulsation mode of Miras (cf. Sect. 3).

- The Iowa models (Bowen [4]; see also Willson [30]) explore the effects of non-LTE cooling on mass loss, describing how thermal winds can be driven due to a 'calorisphere', i.e. a region heated by shock waves and kept warm by inefficient radiative cooling. Alternatively, in the case of rapid cooling these models use a parameterised dust opacity to produce outflows.

- The Berlin models (Fleischer et al.[5], Winters et al. [31], Jeong et al. [18]) introduce a detailed time-dependent description of dust formation which results in a non-linear interaction between gas, dust, and radiation, leading to complex structures and variability. The models are mainly applied to

\footnotetext{
${ }^{1}$ A general description of the relevant physical background can be found, e.g., in Gustafsson \& Höfner ([9]).
} 
determine mass loss rates and other wind properties, especially for cool, dust-enshrouded stars.

- The Vienna models (Höfner \& Dorfi [14], Höfner et al. [16], [15]) include a similar detailed description of dust formation for C-rich chemistry, and they combine time-dependent hydrodynamics with frequency-dependent radiative transfer. The latter allows to take the effects of molecular line blanketing properly into account, leading to more realistic atmospheric structures as demonstrated by comparison of synthetic spectra with observations (cf. Sect. 3).

An overview of dynamical models, including a detailed comparison of input physics, is given in the recent review by Woitke ([33]). The application of wind models to stellar evolution is discussed by Willson ([30]).

\section{Infrared spectra: models meet observations}

When modelling the infrared spectra of cool giants, there are two basic philosophies. One approach, usually driven by new observational data, is semi-empirical modelling of specific observable properties of given stars. Examples are semiempirical models for molecular line profiles which allow to deduce information about the velocity structure of atmospheres and winds (e.g. Keady et al. [22], Keady \& Ridgway [23]) or 'composite' models of the stellar photosphere and circumstellar material for the interpretation of ISO spectra, e.g. 'slab' models of levitated warm molecular gas (e.g. Yamamura et al. [36], Matsuura et al. [26]).

The other method, often motivated by theoretical interests, is the construction of more generally applicable self-consistent models, and iterative improvement of these models by comparison with observations. The models discussed in Sect. 2 fall into this second category: they aim at describing the time-dependent structure of the atmosphere of AGB stars - and in many cases the mass loss through a stellar wind - from first principles. In a second step, snapshots of these structures are used as input for detailed radiative transfer calculations, yielding synthetic spectra and other observable properties (colours, monochromatic radii, etc.) which can be compared to observations.

\subsection{Consistency, all the way ?}

Considering the issue of consistency, it is, in principle, not sufficient if the structure of the model is calculated in a self-consistent way. The a posteriori computation of observable properties has to be consistent with the assumptions and micro-physical data used in the dynamical model. In the ideal case (which is often realized in classical static atmospheres) the calculation of synthetic spectra should just be a re-computation of the radiative transfer as in the original model but with a higher spectral resolution.

In practice, however, there are different degrees of inconsistency between physical assumptions made when calculating model structures or synthetic spectra, and it depends on the problem under investigation what is acceptable and 
what is not. For example, when studying specific spectral lines which would not make a noticeable contribution to the total opacity, and therefore not influence the global energy and momentum balance of the model, it may be sufficient to introduce these lines only in the detailed radiative transfer. On the other hand, strong opacity sources in wavelength ranges with high flux (e.g. IR bands of abundant molecules like water vapour) may influence the structure of the model considerably, and it is essential to use the same opacity data both in the dynamical calculation and in the computation of the observable properties (see contribution by Aringer et al., this volume).

An even more problematic issue is the use of grey opacities in the calculation of the model structures, as is often done to save computing time in dynamical models. This may lead to unrealistic density-temperature structures, a problem that has sometimes been circumvented by only using the density and velocity from the dynamical model, and then recalculating the temperature for the computation of the spectra (e.g. Bessel et al. [3]).

Neglecting the effects of velocity fields on spectral lines when evaluating the opacities during dynamical calculations may be a borderline case of acceptable inconsistency: in contrast to, e.g., line-driven winds of hot stars, the Doppler shifts are generally small in cool giants, and the molecular line blanketing probably makes the effect even less pronounced. Other sources of uncertainty in this context are non-LTE effects (due to shocks), chemical non-equilibrium, or convective motions, which are not included in standard dynamical model atmospheres. At present, it is difficult to estimate which influence these phenomena have on the structures of cool atmospheres and winds.

\subsection{Crucial tests for dynamical models}

Before dynamical model atmospheres can be applied as a tool for the interpretation of observations, they have to be subjected to a number of tests which help to evaluate their consistency and reliability. The most simple check is, of course, a comparison of the hydrostatic limit case with classical model atmospheres. Testing of the full dynamical models, however, has to be done against other dynamical models and observations. In the latter case, it is important to pick a number of criteria which check different aspects of the models:

- The global energy distribution is usually a good indicator for the overall structure since different parts of the spectrum are generated in different layers. Therefore, photometric colours and low-resolution spectra which cover a wide wavelength range are important for gauging the models.

- High-resolution spectra test both the structure and dynamics of the extended atmosphere and wind acceleration region. They make a quantitative determination of velocities possible and allow to distinguish between absorption and emission components in crowded molecular bands.

- Time series of observations resulting from monitoring of targets give insights into time-dependent processes and the global dynamics which cannot be extracted from individual spectra. They are both the most crucial test and the most promising application for dynamical models. 
Another important aspect is that these various criteria have to be fulfilled simultaneously, i.e. a sequence of snapshots from one dynamical model has to fit all the available data for a given star, in order to claim that the model is consistent and realistic.

The models currently found in the literature usually only match part of the criteria given above, indicating that they are probably still too specialised on certain aspects and do not take all necessary processes properly into consideration. In the following sections we will look at examples, focusing on the infrared part of the spectrum.

\subsection{Low-resolution spectra - the global picture}

The spectrometers of the Infrared Space Observatory (ISO) opened possibilities to obtain a simultaneous coverage of a wide wavelength range, including spectral regions not accessible from the ground. This provided a unique way of studying the global structures of atmospheres and inner wind regions of AGB stars and lead to a renewed interest in the modelling of infrared spectra of these stars. After an initial phase dominated by semi-empirical modelling (e.g. Yamamura et al. [36], Matsuura et al. [26]), studies based on reasonably realistic, self-consistent dynamical models are beginning to emerge, both for the interpretation of ISOSWS data, and ground-based observations.

Tej et al. ([29]) discuss synthetic optical and NIR spectra resulting form the dynamical models of Bessell et al. ([3]) and Hofmann et al. ([12]). They find reasonable agreement of the overall energy distribution between 0.5 and $2.5 \mu \mathrm{m}$ with spectra obtained by Lançon \& Wood [24]. They do not, however, attempt to identify a single 'best fit' dynamical model for each star, since the sample of dynamical models is small and various spectra from different models may reproduce the observed spectra equally well. Furthermore, they discuss the problem of fitting the region around $1 \mu \mathrm{m}$ which can possibly be attributed to missing or incorrect molecular opacities.

Hron et al. ([17]) presented a first comparison of ISO-SWS spectra of $\mathrm{R}$ Scl with synthetic spectra based on the dynamical models of Höfner et al. ([16]). While these (grey) models were still too crude to allow for a quantitative fit, they could reproduce the variation of molecular features qualitatively. Since then, the dynamical models have been improved by including non-grey radiative transfer, which turned out to be crucial for obtaining more realistic structures (Höfner [13], Höfner et al. [15]). The observable properties resulting from this new generation of dynamical models show a dramatic improvement compared to earlier grey models (see, e.g., Aringer et al. [2], Andersen et al. [1]).

Recently, Gautschy-Loidl et al. ([8]) have performed a systematic comparison of spectra resulting from the non-grey models of Höfner et al. ([15]) with observations of the C-rich AGB stars TX Psc, WZ Cas, V460 Cyg, T Lyr and S Cep (ISO spectra, spectra from KAO and ground-based observations, narrow-band colours between 0.5 and $1.1 \mu \mathrm{m}$ ). In the wavelength range between 0.5 and $5 \mu \mathrm{m}$, they find good agreement between observations at different phases and a single model for each star. Figure 1 shows a comparison of observational data for TX 

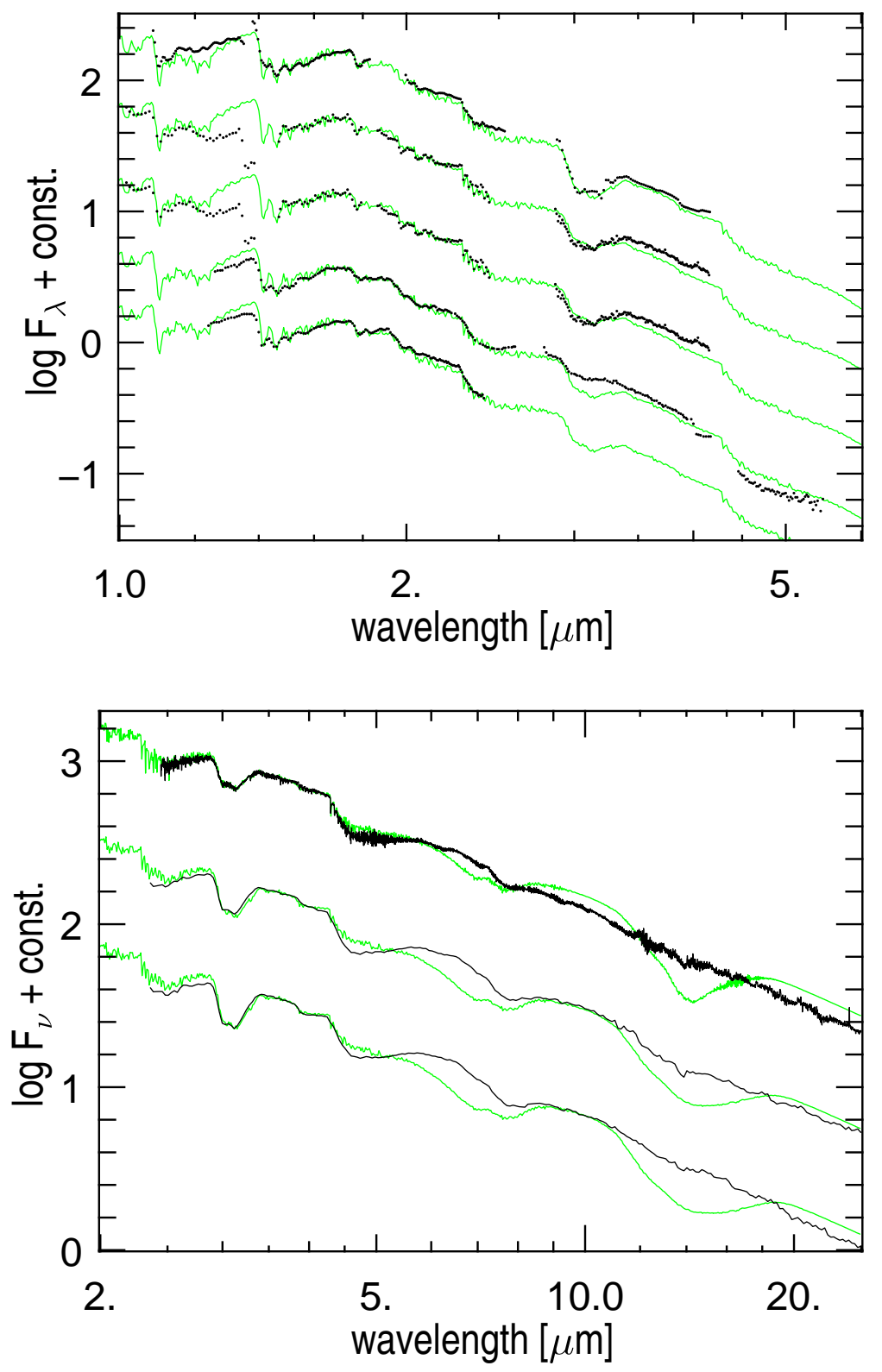

Fig. 1. Synthetic spectra (grey) and observational data of TX Psc (black; upper panel: data from Joyce [20], Lázaro et al. [25] and two KAO spectra; lower panel: ISO SWS06 and ISO SWS01 spectra); from Gautschy-Loidl et al. ([8]). 


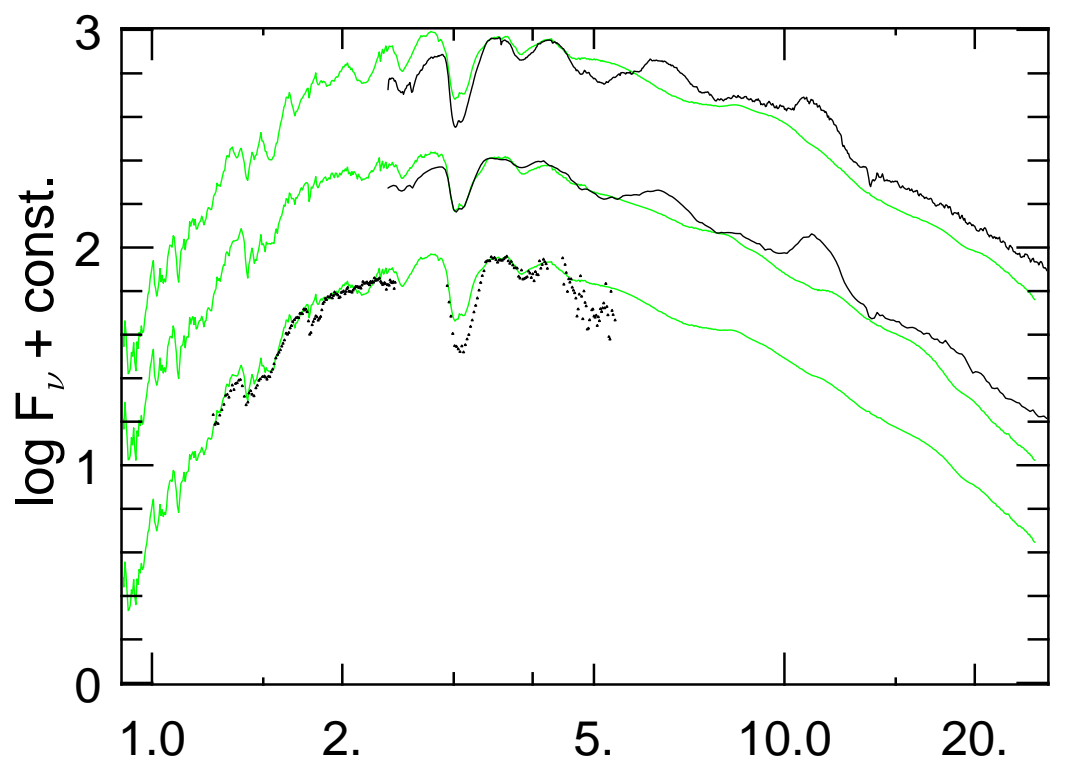

Fig. 2. Synthetic spectra (grey) and observational data of S Cep (black; two ISO SWS01 spectra and one KAO spectrum); from Gautschy-Loidl et al. ([8]).

Psc from various sources with synthetic spectra based on a single dynamical model. The 1-5 $\mu \mathrm{m}$ range (upper panel) is well reproduced but there is an interesting discrepancy between 10 and $20 \mu \mathrm{m}$ (lower panel): the synthetic spectra of the pulsating but dust- and windless model show a pronounced absorption feature around $14 \mu \mathrm{m}$ which is not seen in the observations. Similar problems have been encountered previously when fitting hydrostatic model atmospheres to observed spectra of C-rich AGB stars (e.g. Jørgensen et al. [19]).

While the opposite (i.e. a missing feature in the synthetic spectrum relative to the observed) is easily explained with incomplete opacity data, the appearance of an additional absorption feature in the synthetic spectra is more astonishing, and several attempts have been made to explain this phenomenon (cf. Jørgensen et al. [19]). As discussed by Gautschy-Loidl et al. ([8]), however, dynamical models which show mass loss by stellar winds offer a first self-consistent explanation: Figure 2 compares observed spectra of the Mira variable S Cep with a dynamical model that has a noticeable mass loss by a dust-driven stellar wind. In this case, the synthetic low-resolution spectra show no absorption feature around $14 \mu \mathrm{m}$, since the photospheric absorption feature is filled in by emission from circumstellar material.

This problem of the 'missing' $14 \mu \mathrm{m}$ feature in C-rich AGB stars illustrates nicely how high-resolution spectra can help to clarify a fundamental question: is the feature really absent (which means that there is a major problem with opacities and the model structures, cf. Jørgensen et al. [19]), or is it filled in by 
emission from gas layers above the stellar photosphere, as suggested by dynamical models ?

\subsection{High-resolution spectra - atmospheric dynamics}

High-resolution spectra of AGB stars showing the variation of molecular line profiles with phase are an important tool for studying the dynamics of the pulsating atmospheres and wind acceleration regions (cf. Lebzelter, this volume, for an overview). In particular, vibration-rotation lines of CO in the NIR have been used intensively for temporal monitoring of long-period variables (e.g. Hinkle et al. [10]). The $\mathrm{CO}$ molecule has several advantages over other species: it is abundant, exists both in M- and C-type stars, forms at relatively high temperatures deep in the atmosphere, and stays chemically inert over a wide radial range. Lines corresponding to different transitions are formed in different layers, allowing to probe the velocity structure from the photosphere out into the wind region with just one molecule.

The interpretation of the observations, however, and in particular the derivation of absolute velocities is a non-trivial process which requires the computation of synthetic lines from model structures, and comparison with the observations. Early attempts using semi-empirical models have been mentioned above (Keady et al. [22], Keady \& Ridgway [23]). Here, we discuss a few recent examples based on self-consistent dynamical models.

Winters et al. ([32]) studied synthetic CO fundamental and first overtone lines based on dust-driven wind models and compared these results to observations of the extreme C-rich Mira IRC+10216. The CO fundamental lines at $4.6 \mu \mathrm{m}$ are formed in the zone where the wind has more or less reached its final outflow velocity, showing strong $\mathrm{P}$ Cygni profiles and little temporal variation. In contrast, the first overtone lines at $2.3 \mu \mathrm{m}$ probe the dust formation and wind acceleration region, and the authors interpret the observed variations in terms of shocks and the formation of new dust shells. They discuss the problem of getting a consistent fit for the strengths and shapes of the lines which indicates that the mass loss rate of the model is too high by about a factor of 3 while the dust distribution seems consistent with observations.

Scholz \& Wood ([28]) calculated synthetic CO first and second overtone lines and $\mathrm{OH}$ first overtone lines based on the dynamical model atmospheres by Bessell et al. ([3]) and Hofmann et al. ([12]). They derived conversion factors which relate velocities obtained from the Doppler profiles to the actual radial velocities directly above and below the shock waves in the models. Applying these correction factors to observed velocities they conclude that Mira variables are fundamental mode pulsators.

Nowotny et al. (in prep.) computed synthetic line profiles of $\mathrm{CO}$ fundamental, first and second overtone lines, as well as CN 0-2 red bands (at $2 \mu \mathrm{m}$ ) using dynamical models of Höfner et al. ([15]). The preliminary results show qualitative agreement with observations, e.g., reproducing the S-shaped radial velocity curves observed for CO second overtone lines (cf. Nowotny et al., this volume) 
and $\mathrm{CN}$, including line doubling. The amplitudes of the variations obtained with the models, however, are smaller than the observed variations.

\section{Current projects and trends}

In the previous section we have emphasised the importance of self-consistency when modelling the structures and spectra of cool dynamical atmospheres. We have mentioned a few problems of current models, such as that certain microphysical processes which may be relevant for obtaining a complete and realistic picture are neglected for computational reasons. Apart from these 'intrinsic' problems, there are also a number of open issues concerning parameters and boundary conditions, as well as the overall geometry of the models.

In contrast to classical stellar atmospheres which are determined by three stellar parameters (e.g. mass, luminosity, effective temperature) and the abundances of the chemical elements, dynamical models usually have additional parameters describing stellar pulsation in terms of a variable inner boundary condition (see Sect. 2). There is a lack of suitable pulsation models which could give relations between the basic stellar parameters and quantities describing the pulsation, and sometimes observed period-luminosity relations are used instead. The ultimate goal, however, should be models that describe both the pulsation and the atmosphere and wind in a consistent way. A first step in this direction has been taken by Bessell et al. ([3]) and Hofmann et al. ([12]) who used pulsation models to deduce boundary conditions for atmospheric models. The model atmospheres are a 're-computation' with higher spatial resolution and more complex micro-physics of the outermost layers of the pulsation models.

A common assumption in all models discussed so far is spherical symmetry. This assumption needs to be tested in the light of interferometric observations which seem to indicate non-spherical stellar shapes or giant spots in the photospheres of AGB stars (e.g. Karovska et al. [21], Hofmann et al. [11]; see Scholz [27] for a recent review on interferometry). Woitke et al. ([34]) investigated a possible instability of dust formation in a non-homogeneous stellar atmosphere. This instability is caused by shadows which are cast by regions with a higher degree of condensation (and therefore higher optical depth), improving the conditions for dust formation in the regions which are shielded from the stellar radiation.

Large-scale convective motions are likely to cause inhomogeneities in the atmospheres of cool giants. Freytag succeeded in applying three-dimensional radiation-hydrodynamical 'star-in-a-box' simulations to supergiants (Freytag [6]) and to AGB stars, demonstrating the presence of giant convections cells (Freytag \& Höfner [7]). Recently, Freytag \& Höfner included a description of timedependent dust formation into the $\mathrm{CO}^{5} \mathrm{BOLD}$ code to test the influence of these giant convection cells on grain formation. The formation and growth of dust grains in atmospheres of AGB stars proceeds far from equilibrium, with temperature acting as a threshold and densities of condensible material determining the efficiency of grain growth. Therefore, one may expect that the inhomogeneities in the density and temperature which are caused by the convection cells will be 
$3.47 \mathrm{yrs}$

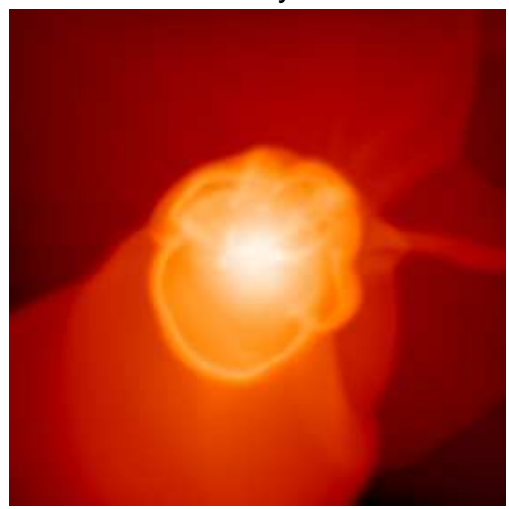

3.87 yrs

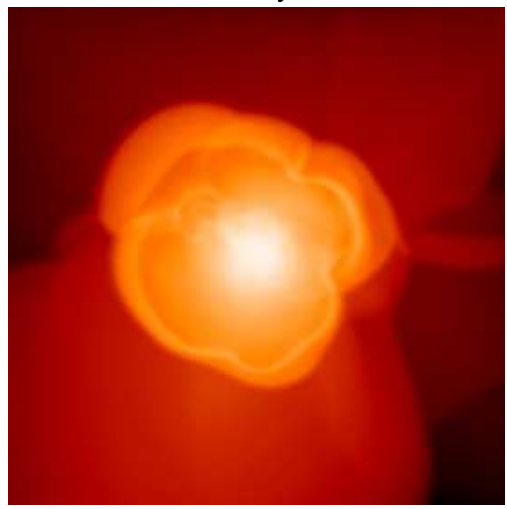

$4.26 \mathrm{yrs}$

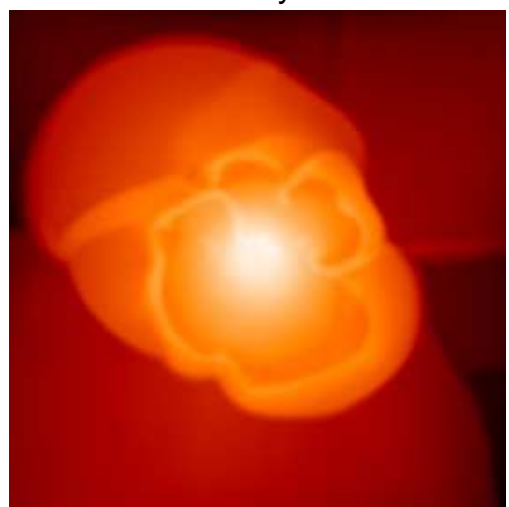

$3.47 \mathrm{yrs}$

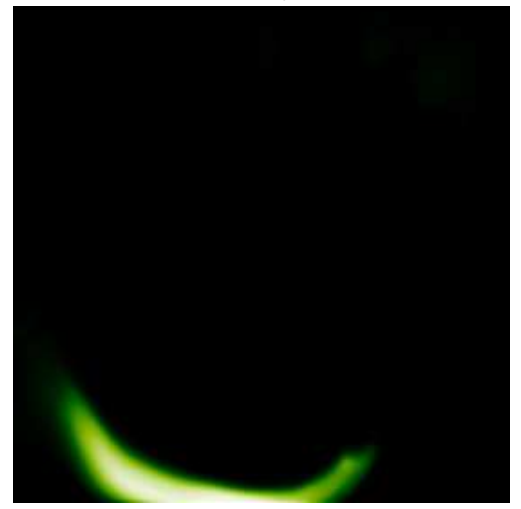

$3.87 \mathrm{yrs}$

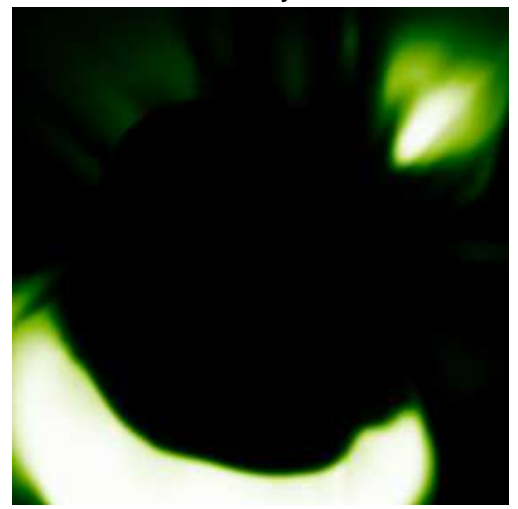

$4.26 \mathrm{yrs}$

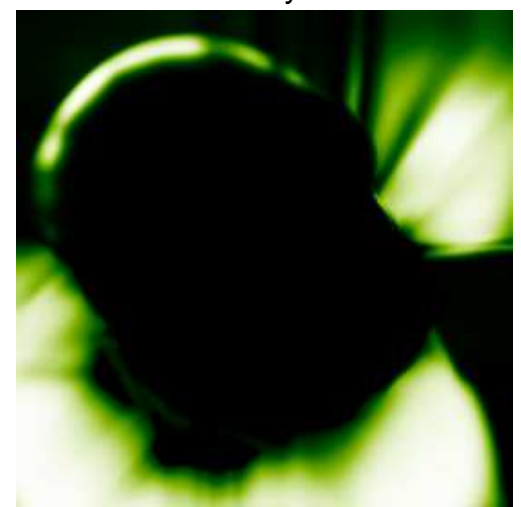

Fig. 3. Cut through the center of a 3D radiation-hydrodynamical model ('star in a box') at three instants; left column: gas density; right column: dust-to-gas ratio (dark colours indicate low values, light colours high values; the 'stellar surface' corresponds approximately to the strong density gradient contour about halfway from the center of the box, just above the density inversion). The calculation was performed using $171 \times 171 \times 171$ grid points. 
imprinted on the dust distribution around the star. Preliminary results seem to confirm this expectation: Figure 3 shows a cut through the center of a 3D model at three instants (left column: gas density; right column: dust-to-gas ratio). The top panels show the model shortly after the equations describing dust formation have been switched on. The condensation process is fastest in the dense, cool gas in the lower left corner in the wake of a shock. Gradually, dust grains form and grow in somewhat less dense regions (middle panels) and in the wake of a new shock wave (upper left corner of the bottom panels). Note the rather sharp inner edge of the dust distribution due to the high temperatures close to the star.

\section{Conclusions}

Since the beginnings of time-dependent dynamical modelling more than two decades ago, dynamic model atmospheres for cool giant stars have overcome many physical and computational obstacles. Within the last few years a transition from qualitative to consistent quantitative modelling is taking place. Current models include detailed micro-physics and some non-equilibrium processes in the computations of atmospheric structures and stellar winds. The resulting synthetic spectra compare reasonably well with observations, but certain consistency issues remain unsolved so far. This has to be kept in mind when using dynamical models for the interpretation of observations.

In addition to the spherical models with detailed input physics, first prototypes of global 3D radiation-hydrodynamical models ('star-in-a-box') have been computed recently, investigating the dynamics of giant convection cells and their influence on atmospheric structure and dust formation. These 3D models are based on simpler micro-physics and have a lower spatial resolutions than the spherical models. This trade-off is necessary to keep computation times within acceptable limits.

In summary, the present status of dynamic model atmospheres and the developments that can be expected within the next few years look promising, regarding the interpretation of high-resolution IR spectra.

\section{References}

1. Andersen A.C., Höfner S., Gautschy-Loidl R. 2003, A\&A 400, 981

2. Aringer, B., Jørgensen, U.G., Kerschbaum, F., Hron, J., \& Höfner, S. 2002, in: Radial and Nonradial Pulsations as Probes of Stellar Physics, Aerts, C., Bedding, T., Christensen-Dalsgaard, J. (eds.), A.S.P. Conf. Ser. 259, p. 538

3. Bessell M.S., Scholz M., Wood P.R. 1996, A\&A 307, 481

4. Bowen G.H. 1988, ApJ 329, 299

5. Fleischer A.J., Gauger A., Sedlmayr E. 1992, A\&A 266, 321

6. Freytag B. 2003, in Interferometry for Optical Astronomy II, Traub W.A. (ed.), Proc. of SPIE, Vol. 4838, p. 348

7. Freytag B., Höfner S. 2003, AN 324, Suppl. Issue 3, p. 173 
8. Gautschy-Loidl R., Höfner S., Jørgensen U.G., Hron J. 2004, A\&A, submitted

9. Gustafsson B., Höfner S. 2004, in: Asymptotic Giant Branch Stars, Habing H.J., Olofsson H. (eds.), Springer, p.149

10. Hinkle K.H., Hall D.N.B., Ridgway S.T. 1982, ApJ 252, 697

11. Hofmann K.-H., Balega Y., Scholz M., Weigelt G. 2000, A\&A 353, 1016

12. Hofmann K.-H., Scholz M., Wood P.R. 1998, A\&A 339, 846

13. Höfner S. 1999, A\&A 346, L9

14. Höfner S., Dorfi E.A. 1997, A\&A 319, 648

15. Höfner S., Gautschy-Loidl R., Aringer B., Jørgensen U.G. 2003, A\&A 399, 589

16. Höfner S., Jørgensen U.G., Loidl R., Aringer B. 1998, A\&A 340, 497

17. Hron J., Loidl R., Höfner S. et al. 1998, A\&A 335, L69

18. Jeong K. S., Winters J. M., Le Bertre T., Sedlmayr E. 2003, A\&A 407, 191

19. Jørgensen U.G., Hron J., Loidl R. 2000, A\&A 356, 253

20. Joyce R.R. 1998, AJ 115, 2059

21. Karovska M., Nisenson P., Papaliolios C., Boyle R. 1991, ApJ 374, L51

22. Keady J.J., Hall D.N.B., Ridgway S.T. 1988, ApJ 326, 832

23. Keady J.J., Ridgway S.T. 1993, ApJ 406, 199

24. Lançon A., Wood P.R. 2000, A\&AS 146, 217

25. Lázaro C., Hammersley P.L., Clegg R.E.S., et al. 1994, MNRAS 269, 365

26. Matsuura M., Yamamura I., Cami J., Onaka T., Murakami H. 2002 A\&A 383, 972

27. Scholz M. 2003, in: Interferometry for Optical Astronomy II, Traub W.A. (ed.), Proc. SPIE, Vol. 4838, p. 163.

28. Scholz M., Wood P.R. 2000, A\&A 362, 1065

29. Tej A., Lançon A., Scholz M., Wood P.R. 2003, A\&A 412, 481

30. Willson L.A. 2000, ARAA 38, 573

31. Winters J.M., Le Bertre T., Jeong, K.S., Helling C., Sedlmayr E. 2000, A\&A 361, 641

32. Winters J.M., Keady J.J., Gauger A., Sada P.V. 2000, A\&A 359, 651

33. Woitke P. 2003, in: Modelling of Stellar Atmospheres, IAU Symposium, Vol. 210, Piskunov N. E., Weiss W. W., Gray D.F. (eds.), p. 387

34. Woitke P., Sedlmayr E., Lopez B. 2000, A\&A 358, 665

35. Wood P.R. 1979, ApJ 227, 220

36. Yamamura I., de Jong T., Cami J. 1999, A\&A 348, L55 